\title{
EDITORIAL Biomarkers: stepping stones to firmer end points
}

\author{
Journal of Human Hypertension (2016) 30, 413; doi:10.1038/ \\ jhh.2016.33
}

Encompassing a diverse array of parameters, biological markers (or biomarkers) have been defined by the Biomarkers Definitions Working Group of the National Institutes of Health as 'A characteristic that is objectively measured and evaluated as an indicator of normal biological processes, pathogenic processes, or pharmacologic responses to a therapeutic intervention.. ${ }^{1}$ As such, biomarkers include a wide range of variables derived from history taking, physical examination, analysis of fluids and tissues, medical imaging, electrocardiographic and other forms of neural and muscular assessment, functional testing, and many other forms of physiological and pathological analysis.

Biomarkers can serve a number of different roles:

- As surrogate end points, they can substitute for clinical outcomes (such as myocardial infarction, stroke or survival). Common examples include the assessment of therapeutic interventions (such as drugs, surgery, devices, intraluminal procedures, vaccines and biological agents) and of the natural history or epidemiological studies of various disease states. Blood pressure is used as a biomarker to study the therapeutic efficacy of potential new antihypertensive agents aimed at reducing the risk of complications such as stroke. In this role, measurement of biomarkers can be more efficient, less expensive or more feasible when direct measurement of the clinical end points of interest would require an unacceptable amount of time, resources or number of patients to draw statistically sound conclusions.

- As diagnostic tools, biomarkers can provide information that increases or decreases the likelihood of a disease state being present (for example, the measurement of ferritin in exploring the possibility of haemochromatosis).

- As staging tools, biomarkers can help to indicate the severity or extent of progression of a disease state (for example, measurement of estimated glomerular filtration rate in chronic kidney disease, or extent of tumour spread as defined by imaging studies).

- As prognostic indicators, they can help predict the course of a medical condition (for example, falls in tumour markers following medical or surgical treatment).

- They can also be used in prediction tools and for monitoring responses to treatment (for example, measurement of serum cholesterol as a component of a prediction calculator for future cardiovascular events and in response to statin therapy).

While they have great potential to provide valuable information, biomarkers are not without shortcomings. With regard to therapeutics, an effect of a treatment modality on a biomarker does not necessarily prove an effect on a clinical outcome, as was demonstrated by the results of the Cardiac Arrhythmia Suppression Trial, in which rates of death due to arrhythmia or cardiac shock following myocardial infarction among patients receiving active treatment designed to suppress arrhythmias were in fact higher than those receiving placebo. ${ }^{2}$ Conversely, the lack of an effect on a biomarker does not necessarily mean that the treatment will not have a beneficial effect on clinical outcomes. Hence, authorities urge that biomarkers should not serve as the sole basis for the introduction of novel therapies, but may form part of the provisional evaluation process, following which confirmation of clinical efficacy and safety should involve assessment of hard clinical end points before the treatment is considered acceptable for clinical application. ${ }^{1,3}$

The value of a biomarker depends critically on the care with which it is measured, the degree of standardization of measurement conditions, and the extent to which confounders are controlled and patient/control groups matched. With recent trends moving towards the use of high-throughput technologies to characterize disease states on the basis of their genomic, proteomic and metabolomic signatures, there is a concern that it will simply not be feasible to apply such rigour to the enormous number of variables that will be assessed in this way. Analysing such complex massive data sets also poses challenges for workers in this field.

The current special edition of the Journal of Human Hypertension focusses on the use of biomarkers in the evaluation of various hypertension-related states. With the exception of one study that assessed measures of small- and large-artery function, all examined the use of circulating biomarkers, some of them familiar (including uric acid, advanced glycation end products, homocysteine and sodium) and others not so much (including pentraxin 3, neopterin and desphospho-uncarboxylated matrix GLA protein). They also share a focus on associations of these biomarkers either with other clinical parameters (such as blood pressure, vascular stiffness, endothelial function, components of the metabolic syndrome or other circulating biomarkers) or with clinical outcomes (cardiovascular events in patients with chronic kidney disease).

These studies demonstrate the ability of biomarkers to inform on potential pathogenetic mechanisms and to predict cardiovascular outcomes and responses to therapeutic interventions. As such, each has a chance of ultimately becoming introduced into routine clinical practice, but not before extensive further studies confirm their validity, reproducibility and applicability to the clinical setting.

\section{CONFLICT OF INTEREST}

The author declares no conflict of interest.

Michael Stowasser Endocrine Hypertension Research Centre, University of Queensland School of Medicine, Greenslopes and Princess Alexandra Hospitals, Brisbane, QLD, Australia E-mail: m.stowasser@uq.edu.au

\section{REFERENCES}

1 Biomarkers Definitions Working G. Biomarkers and surrogate endpoints: preferred definitions and conceptual framework. Clin Pharmacol Ther 2001; 69: 89-95.

2 Echt DS, Liebson PR, Mitchell LB, Peters RW, Obias-Manno D, Barker AH et al. Mortality and morbidity in patients receiving encainide, flecainide, or placebo. The Cardiac Arrhythmia Suppression Trial. N Engl J Med 1991; 324: 781-788.

3 Psaty BM, Weiss NS, Furberg CD, Koepsell TD, Siscovick DS, Rosendaal FR et al. Surrogate end points, health outcomes, and the drug-approval process for the treatment of risk factors for cardiovascular disease. JAMA 1999; 282: 786-790. 